\title{
Relative enrichment - a density-based colocalization measure for single- molecule localization microscopy
}

Aske L. Ejdrup ${ }^{1,2}$, Matthew D. Lycas ${ }^{1}$, Niels Lorenzen ${ }^{1}$, Ainoa Konomi ${ }^{1}$, Freja Herborg ${ }^{1}$, Kenneth L. Madsen ${ }^{1}$ and Ulrik Gether ${ }^{1,2}$

${ }^{1}$ Department of Neuroscience, Faculty of Health and Medical Sciences, University of Copenhagen, Denmark

${ }^{2}$ Correspondance: ejdrup@sund.ku.dk or gether@sund.ku.dk 


\section{Abstract}

2 Dual-color single-molecule localization microscopy (SMLM) provides unprecedented

3 possibilities for detailed studies of colocalization of different molecular species in a cell.

4 However, the informational richness of the data is not fully exploited by current analysis tools

5 that often reduce colocalization to a single value. Here, we describe a new tool specifically

6 designed for determination of co-localization in both 2D and 3D from SMLM data. The approach

7 uses a novel function that describes the relative enrichment of one molecular species on the

8 density distribution of a reference species. The function reframes the question of colocalization

9 by providing a density-context relevant to multiple biological questions. Moreover, the function

10 visualize enrichment (i.e. colocalization) directly in the images for easy interpretation. We

11 demonstrate the approach's functionality on both simulated data and cultured neurons, and

12 compare it to current alternative measures. The method is available in a Python function for

13 easy and parameter-free implementation. 


\section{Introduction}

16 Fluorescence light microscopy (LM), combined with immuno-cyto- or -histochemistry, has been

17 used extensively for studies of colocalization of different cellular components. These

18 experiments have been central to our understanding of the cell, where function of a molecular

19 species is partially inferred from spatial association to a reference protein or organelle.

20 Accordingly, a host of methods for quantitative colocalization analysis for wide-field image

21 application have been developed [1]. Common to these is the conceptual reduction of

22 colocalization to a single value. This has been a rational approach, given that the $\sim 250 \mathrm{~nm}$

23 diffraction-limit of light is orders of magnitude larger than individual molecules [2]. With the

24 advance of novel single-molecule localization microscopy (SMLM) techniques, molecular

25 positions can be resolved down to $<20 \mathrm{~nm}$ by temporally separating spatially overlapping light

26 sources [3-5]. With the resolution approaching molecular sizes scientists have been provided

27 with a tool to study nanoarchitecture and carry out more detailed colocalization studies.

28 But no new venture is without new woes. SMLM techniques produce coordinate-based

29 molecular positions, rather than the intensity-based pixel information of regular LM. This

30 change in data type makes conventional analyses difficult to apply. Alongside, non-uniform

31 protein nanoscale distribution in cellular membranes and cytoplasm is becoming increasingly

32 apparent [6, 7], which makes it relevant to assess colocalization in the context of protein

33 density. Lastly, as molecular localization-precision is approaching that of individual molecule

34 size, and certainly within the size of most molecular labels, a simplistic view on colocalization is

35 arguably a remnant from the diffraction-limit-derived conceptual reduction of the 
phenomenon. By expanding the colocalization analysis to include biologically relevant context,

37 the data might further address emerging scientific questions.

While several methods have been developed to assess colocalization in super-resolved

39 microscopy data, they either reduce the measure to a single value, which may limit biological

40 insight, or depend on user-defined input parameters. Rossy and colleagues [8], coordinate-

41 based colocalization (CBC) [9] and cluster detection with degree of colocalization (ClusDoC) [10]

42 all employ parameter-based approaches where user-defined values strongly influence

43 outcome, [11] and neither ClusDoC nor Rossy and colleagues approach work with 3D data.

44 While $\mathrm{CBC}$ does include a density context in its calculations, the resulting value incorporate

45 local density of both species and compress the information to a summary statistic between -1

46 and 1 that provides rather little information on where the interaction takes place. Coloc-

47 Tesseler [12] works in 3D and is density based, but likewise it outputs a summary statistic that

48 reduces colocalization to a single value without further context to the value.

In this work, we present a novel function that describes relative enrichment of one

50 molecular species on the density distribution of a reference species. The algorithm is inspired

51 by the pair-correlation function [13] and the Voronoï tessellation of Coloc-Tesseler [12]. It

52 reframes the question of colocalization by providing a density-context relevant to numerous

53 biological questions. The tool is parameter free and outputs a unitless ratio measure based on

54 the average distance to neighboring proteins. Further, the algorithm can visualize enrichment

55 directly in the images for qualitative interpretation, making colocalization information from

56 super resolution microscopy interpretable by scientists without advanced microscopy expertise.

57 It works in both 2D and 3D and is available as a python function for further open development 
58 and easy implementation in a processing pipeline across operating systems and file formats.

59 We envision this tool broadly used in future studies of cellular nano-architectural organization

60 due to its intuitive, unitless representation of colocalization, its contextual insight when protein

61 distribution is heterogenous, its ability to generate informative qualitative images, and its easy

62 and parameter-free implementation. 


\section{Results}

\section{Tessellation-based assessment of relative enrichment}

65 Voronoï tessellation partitions a plane into discrete regions around seeds with each region

66 containing the area closest to its seed (Fig. 1). The method can be applied to SMLM data, where

67 each molecular localization represents a seed, and has proven itself a robust and efficient tool

68 to assess local protein density [14-16]. Previously, Levet and colleagues proposed a

69 colocalization analysis based on tessellation [12]. Building on their approach, we propose an

70 integration of tessellation and conceptual elements of the pair-correlation function, with the

71 aim to unfold new biological insight by deriving a continuous measure of colocalization in the

72 context of density.

73 Our method assesses the enrichment of one molecular species, referred to as the

74 primary species, across the density distribution of a reference species. The result is a density

75 plot of the reference species, with a score indicating how enriched the primary species is in

76 areas of differing densities of the reference. The two species can be interchanged depending on

77 the biology of interest.

To illustrate the method, we simulated two randomly distributed species of localizations

79 (Fig. 1A). First step is to split the assigned reference species into separate regions by Voronoï

80 tessellation (Fig. 1B). We refer to these as reference regions. Regions with no outer neighbor

81 are considered edge regions, denoted with dashed lines and are not included in the analysis.

82 Localizations of the primary species are then superimposed in their original positions (Fig. 1C),

83 and the heart of the method is to quantify the observed number of primary localizations for

84 each reference region, and compare it to the expected amount: 
Eq. 1 relative enrichment $=\frac{\text { primary }^{\text {observed }}}{\text { primary }^{\text {expected }}}$

85 The expected number of primary localizations is calculated for each region as per Eq. 2, by

86 dividing the individual region area with the total area of all regions, and then multiplying that

87 number with the total number of observed primary localization:

Eq. $2 \quad$ primary $y^{\text {expected }}=\frac{\text { area }^{\text {region }}}{\text { area }^{\text {total }}}$ primary $^{\text {total }}$

88 To exemplify this, the relative enrichment (RE) is calculated for the region denoted with an

89 asterisk on Fig. 1C (Fig. 1D). This region has an area of $0.24 \mu \mathrm{m}^{2}$, with the total area of all nine

90 included regions at $1.3 \mu \mathrm{m}^{2}$. That is $18.5 \%$ of total area, and with 20 total primary localizations,

91 Eq. 2 tells us that the expected occurrence is 3.7 localizations. As only one primary localization

92 is found inside that region, Eq. 1 gives us a RE score of 0.27. For two completely uniform

93 distributions, each region of the reference species would have an average RE of 1, and as such,

94 an RE below 1 indicates a depletion. Conversely, an RE above 1 indicates an enrichment of the

95 primary species in the vicinity of the associated reference localization. When plotting all the

96 regions of the reference species color-coded by their $\mathrm{RE}$, the heterogenous distribution of the

97 primary species relative to the reference species becomes evident (Fig. 1E). 


\section{Larger dataset and scoring across densities}

100 A typical SMLM image contains on the order of thousands to millions of localizations. To distil

101 information from a wealth of RE scores in a density context, our method bins reference

102 localizations by their region size, and assess mean enrichment score per bin. To demonstrate

103 this, we simulated normally-distributed, overlapping clusters of each species, slightly off-set

104 from one another, and added random background noise (Fig. 2A). The RE score for each region

105 was assessed as described earlier (Fig. 2B), and an illustrative RE-color-coded map of reference

106 regions is shown on Fig. 2C. Following this procedure, we uniformly binned regions by the

107 logarithm of their area. This generates a histogram that visualize the density distribution of the

108 reference species (Fig. 2D). For each bin, we additionally plot the mean RE of the primary

109 species in reference regions of that size interval. This results in a plot that shows the relative

110 enrichment of one molecular species for a given density of a reference molecular species. This

111 presentation of RE by reference region size provides a density context to colocalization, without

112 reducing it to a one-dimensional phenomenon.

113 Binning regions by area is the most apparent choice, as RE is computed from area.

114 However, area is not an immediately intuitive measure [17]. Therefore, we introduce binning by

115 distance to neighbors, which is a more intuitive parameter, directly correlated to the size of the

116 tessellated area. As complex polygons have no direct radius, mean distance to nearest

117 neighbors is an excellent alternative, that provides a one-dimensional measure for local density.

118 Fortunately, nearest-neighbor distance (NND) scales rather robustly with region area (Fig. 2E),

119 with the added advantage of higher resolution in the densest localizations, due to the concave 
120 shape of the correlation. As shown on Fig. 2F, binning by NND rather than area produce a

121 similar result, but with more intuitive units on the $\mathrm{x}$-axis.

122 To show an example with no colocalization, we once again simulated two species with

123 different distributions: one clustered and one uniform (Fig. 2G). The irregular distribution in one

124 species does not distort the result, and this situation still yields an average RE score of 1 across

125 densities (Fig. 2H), as the method accounts for area covered when computing enrichment. This

126 is true regardless of which species is chosen as reference (Fig. S1A). While some of the smaller

127 regions might score high in RE, this is counteracted by a low score for the majority, and the two

128 will even out in the binning when no specific colocalization is present. This is illustrated by the

129 color-coded plot with RE score by region (Fig. 2I). The bimodal distribution of the reference

130 species is also apparent from the histogram of NND, showing the usefulness of mapping

131 densities when assessing colocalization.

132 Coltharp and colleagues point out that careful consideration should be put into the

133 selected region size when assessing spatial organization [18]. This can significantly impact the

134 colocalization score of many methods, and lead to unwarranted conclusions if neglected. While

135 region size can impact absolute RE values, the scoring across density bins in this method carries

136 information about this spatial distribution (Fig. S1B-E), rather than incorrectly influencing a

137 single measure of colocalization. This further highlights the usefulness of the measure. 


\section{Assessing colocalization of synaptic release proteins}

140 Molecular architecture is highly important for the correct functioning of cells. Especially in

141 neuronal synapses, where various proteins coordinate docking, priming and fusion during

142 synaptic vesicle release at astounding precision and speeds [19]. To showcase the insight

143 supplied by applying our relative enrichment measure, we imaged two proteins central to

144 vesicle fusion: mammalian uncoordinated 18 (Munc18-1) and syntaxin-1 (Stx1). Stx1 has

145 multiple functions and is involved in both neuronal maintenance as well as vesicle docking and

146 fusion [20]. In contrast, Munc18-1 exclusively orchestrate the assembly of release machinery

147 [21]. Evidence from previous studies in neuroendocrine cells suggest the two proteins

148 colocalize at docked vesicles and disperse following vesicular neurotransmitter release [22-25].

149 We harvested hippocampal neurons from rat pups, fixated the cultures, stained for the two

150 proteins and imaged with direct stochastic optical reconstruction microscopy (dSTORM) [26]

151 (see methods for detailed protocol). Under basal conditions, the two proteins appear to form

152 nanoclusters (Fig. 3A-B). This is in line with findings showing that both proteins exist in clusters

153 at resting conditions [24, 27]. Next, we assessed colocalization with our proposed method. As

154 relative enrichment can be examined bidirectionally, we alternated the appointed primary and

155 reference species: colocalization of primary Stx1 to reference Munc-18 (Fig. 3C, left) or

156 colocalization of primary Munc-18 to reference Stx1 (Fig. 3C, right). Both species showed a

157 propensity to colocalize with the densest clusters of the opposing species, as maximal

158 enrichment is at localizations with less than $10 \mathrm{~nm}$ on average to adjacent molecules of the

159 same species (Fig. 3D and E). However, the relative enrichment was higher for Munc-18 on

160 Stx1, possibly because Stx1 is in functional excess relative to Munc18-1, as Stx1 has multiple 
161 independent roles in neuronal maintenance and neurotransmitter release [20]. This relative

162 over-enrichment of Munc18-1 on Stx1 as compared to vice-versa can also be illustrated by

163 color-coding the reference species based on individual RE score, as previously shown (Fig. 3F).

164 Here, we plot the individual localizations rather than the associated regions, as the border

165 between extracellular space and cells is harder to distinguish in these more complex neuronal

166 structures when using Voronoï regions (Fig. S2A). Next, we treated the primary hippocampal

167 cultures with N-methyl-D-aspartate (NMDA) to activate NMDA-type ionotropic glutamate

168 receptors and stimulate vesicular neurotransmitter release, or with tetrodotoxin (TTX), a

169 sodium channel blocker, to prevent vesicular release (Fig. 3G). As Munc18-1 was more tightly

170 associated with Stx1 during basal conditions, we focused on this enrichment-directionality.

171 Upon stimulating release with NMDA, enrichment of Munc18-1 on Stx1 dropped in the densest

172 Stx1 localization (Fig. 3H), in line with their proposed role in the synaptic vesicle fusion [22]. In a

173 mirror image, blocking synaptic vesicle release with TTX increased the propensity of the two

174 proteins to colocalize, consistent with an increase in vesicular docking or priming. This effect

175 can again be visualized by plotting the reference species, Stx1, color-coded by RE score (Fig. 3I,

176 Fig. S2B). We observed no significant redistribution of Stx1 after treatment (Fig. S2C), matching

177 previous findings when analyzed with tessellation [28]. But tessellation may not uncover all

178 reorganizational nuances. Results from applying the same analysis with primary and reference

179 species reversed is shown in Fig. S2D, E.

180 Lastly, we compared our results to the other tessellation-based method, Coloc-Tesseler

181 [12]. This method calculates either Spearman or Mander's coefficient, and while both can be

182 computed in two ways similarly to our analysis, they are not directly comparable. When 
bioRxiv preprint doi: https://doi.org/10.1101/2022.02.08.479535; this version posted February 9, 2022. The copyright holder for this preprint (which was not certified by peer review) is the author/funder. All rights reserved. No reuse allowed without permission.

183 comparing the coefficient values of NMDA and TTX treated cells (Fig. 3J), neither of the four

184 coefficients showed a significant difference, in contrast to both our proposed method and

185 literature findings.

186 


\section{Validation on a simulated 2D and 3D vesicle example}

188 As membranes are relatively flat on a mesoscopic level, 2D models of plasma membrane-bound

189 proteins, and by extension 2D images, capture a good part of the relevant biology. Some

190 molecules, however, reside in the cytoplasm or on the membrane of different organelles. To

191 fully capture their spatial distribution, analyses need to incorporate z-positions from 3D images.

192 The same is true in thicker tissue samples. To test the 3D capabilities of our method, we

193 simulated vesicles at an active zone. Three different molecular species were simulated: a

194 plasma membrane-bound active-zone-like (AZ) species, i.e. SNAP-25, a species bound to the

195 vesicle membrane, i.e. synaptophysin, and a cargo species in the lumen of the vesicle, i.e.

196 neuropeptides (Fig. 4A). As input to our analysis, we generated ten images with twenty vesicles

197 in each, all of which contained the cargo molecule but only half were docked and associated

198 with a cluster of the AZ molecule (Fig. 4B). As most 3D SMLM techniques have a higher

199 uncertainty in the z-plane, the random jitter representing technical uncertainty was doubled for

200 this axis. To mimic the results that similar biology would yield with 2D SMLM, we projected the

201 simulated 3D images on to a 2D plane (Fig. 4C).

202

When assessed in 2D, using the vesicle species as reference, the $A Z$ and cargo species

203 have equal RE score across the vesicle density distribution (Fig. 3D). They both appeared as

204 highly enriched around the densest vesicle membrane localizations and fell below an RE score

205 of 1 outside of the vesicles, indicating under-enrichment in the surrounding space.

206 In contrast, a different distribution was resolved when analyzed in 3D. Here, a high degree of

207 colocalization between the cargo and vesicles species was still evident, whereas the enrichment

208 of the AZ species was reduced as expected, as apparent from the RE score (Fig. 4E). Based on 
209 the histogram of vesicle NND, we can see a preferential colocalization of the cargo to the

210 dense-most localizations of the vesicle, whereas the AZ species peaks at the intermediate

211 density, suggesting a more peripheral association. This becomes evident when plotting the

212 vesicle localization color-coded for their RE score of the two species (Fig. 4F). The AZ species

213 clearly enriches the bottom portion of vesicles near the membrane, whereas the cargo species

214 highlights the center of all vesicles. This difference is much clearer in 3D as compared to the 2D

215 visualization (Fig. S3A, B). Analysis of these simulated data underscores the importance of a 3D-

216 compatible colocalization analysis for certain biological contexts.

218 Investigating protein trafficking itineraries

219 Protein trafficking is an important element in cell biology, where 3D information is often

220 needed to draw a conclusion. To investigate whether our method can inform us of trafficking

221 itineraries, we tested it on experimental data of endocytosis of two different transmembrane

222 proteins, the dopamine (DAT) and noradrenaline transporter (NET). The two transporters

223 mediate clearance of their respective substrates, dopamine and noradrenaline, and play an

224 important role in shaping the spatiotemporal profile of dopaminergic and noradrenergic

225 neurotransmission [29, 30]. Interestingly, DAT and NET display differences in subcellular

226 localization and trafficking itinerary [28, 31-33]. Specifically, NET has been reported to

227 internalize more rapidly than DAT and preferentially sort to the Rab11 recycling compartment

228 rather than for degradation, whereas the internalized DAT sort less to this compartment.

229 However, the specific path for the two transporters through near-membrane organelles is

230 difficult to assess with conventional LM, due to their proximity to the cell surface. A central 
231 compartment to this near-surface trafficking is the early endosome. These amorphic structures

232 are typically 400-700 $\mathrm{nm}$ in diameter [34], and play a key role in membrane-protein sorting

233 following their internalization [35]. To test if the difference in internalization rates of the two

234 transporters is reflected in the early endosomal steady-state content, we acquired dual-color

235 dSTORM images of the early endosome antigen 1 (EEA1), alongside either anti-DAT or -NET

236 antibodies in PC12 cells. Images for both transporters were captured with astigmatic dSTORM

237 to achieve 3D resolution.

Visualizing the images in 2D from bottom-up by a Gaussian representation of the data,

239 larger EEA1-positive formless structures with various protrusions are visible, matching the size

240 described in the literature (Fig. 5A, B). Likewise, DAT clusters match what has previously been

241 described [36], and NET architecture resembles that of DAT (Fig. 5A, B). Additionally, when

242 assessing depth (z-axis position) of transporters and EEA1-positive structures (Fig. 5C), an

243 intracellular accumulation is present for NET, but not for DAT, matching the expected

244 distribution. We first assessed if a differential colocalization pattern to EEA1 could be resolved

245 for NET and DAT in 2D images. This was done by applying our method without depth (z-

246 coordinates) (Fig. 5D). We used EEA1 as the reference species, and while both transporters

247 showed slight enrichment in the denser endosome localizations, indicating some association,

248 there was no difference between the relative enrichment of NET and DAT in EEA1-positive

249 structures. To assess if 3D information changes the result, we included depth information in the

250 images (Fig. 5E, F, upper panels). Defining plasma membrane depth as median transporter

251 position in the z-axis, EEA1-positive structures were primarily intracellular, but proximal to the

252 surface (Fig. 5C). Additionally, qualitative assessment indicated more NET in these structures. 
253 When plotting EEA1 localization color-coded for their RE-values, the association of DAT and NET

254 to intracellular EEA1 became both qualitatively visible and quantifiable (Fig. 5E, F, lower

255 panels). Particularly for NET. We reassessed relative enrichment by bins in 3D, and from the

256 plot it becomes apparent the densest EEA1 localizations, the putative early endosomes, were

257 significantly more enriched with NET than DAT (Fig. 5G). This provides clear evidence that the

258 higher steady-state internalization rate of NET is, at least in part, mediated by sorting through

259 early endosomes. Utility of the relative enrichment method is underscored when we compare

260 our results to conventional colocalization measures as analyzed by Coloc-Tesseler (Fig. 5H, Fig.

261 S3C). Only one of four measures show significant difference in association the two transporter

262 species to EEA1, and in addition to the question of deciding which measure is appropriate, the

263 single colocalization-value provided with these give no context, compared to the quantitative

264 output and the qualitative visualization of our method. In summary, relative enrichment can

265 contribute with insights into protein trafficking that would otherwise have been difficult, if not

266 impossible, to get otherwise.

267 As a final note, it is worth mentioning that interpretation of the absolute RE score can

268 be more complicated in 3D analyses - particularly when molecules are restricted to certain

269 structures. This is the case for transporter localization to early endosomes, where neither NET

270 nor DAT have free roam of the cytoplasm and must reside in the membrane of organelles. To

271 use the absolute RE values in instances like these, careful thought has to be put into the

272 biology. Here, the relative RE scores between different molecular species is the safer choice.

273 Finally, we once again compared our results to analysis by Coloc-Tesseler (Fig. 5H). 
276 Colocalization analysis is an import tool in LM. Knowing the spatial distribution of a molecule

277 relative to other molecules or compartments in the cell may help infer its function [1]. Indeed,

278 the advent of super resolution microscopy techniques has provided an even greater spatial

279 resolution for this approach. However, new analytical challenges have emerged with these new

280 data. For instance, most of the newly developed methods for colocalization of coordinate-

281 based molecular positions require user-defined input parameters that influence results, and

282 whose impact require in-depth knowledge of the tool to fully grasp [8-11]. Levet and colleagues

283 address that in their two-way tessellation-based approach [12], but like most colocalization

284 analysis tools the final output is reduced to a single value, providing no context or visual

285 representation.

286 In this work, we have presented an alternative approach to colocalization analysis. Here the co-

287 organization of two molecular species is assessed in a density context relevant to a wealth of

288 emerging biological questions, as heterogenous organization of plasma membrane as well as

289 cytoplasmic proteins has become apparent. A parameter free computation of the RE score

290 provides the fold-above-expected occurrence of a primary species, across the density

291 distribution of a reference species. We recommend binning by average distance to nearest

292 neighbor, rather than by region size, as distance is more easily interpretable than area or

293 volume. The method is bilaterally applicable, as the species can be freely interchanged based

294 on the biological question. In addition to the density context provided by the RE plot, the

295 scoring of each individual reference localization allows for semi-quantitative visualization of the

296 co-organization, that can aid deciphering of the underlying biology. The raw data can be 
297 visualized in color-coding based on RE score, with either polygon plots or scatter plots, as

298 shown throughout the study. This visualization tool is a strong hybrid between qualitative and

299 quantitative approaches, that relies on the scoring of each individual localization. It is inspired

300 by Malkusch and colleagues [9], and distinguished the method from ones that are Mander's

301 and Spearman's coefficient-based, where scoring is derived macroscopically.

302 The algorithm is available in Python, rather than as a stand-alone OS-specific software,

303 for easy implementation in an analysis pipeline and future open development. This comes with

304 the added benefit that more and more SMLM-related pre- and post-processing tools are

305 Python-based [37-42]. It is compatible with both 2D and 3D images, and existing Python

306 packages allow for loading of most all conceivable data formats. 


\section{Methods}

\section{Relative Enrichment Function.}

309 First step of calculating relative enrichment (RE) is selecting a reference and a primary species.

310 The reference species then segments the total image $(I)$ into regions by Voronoï tessellation,

311 where $R$ denote the full set of indices belonging to the elected reference species and $\left(L_{r}\right)_{r \in R}$ is

312 each individual localization in the set $R$. Each $L_{r}$ segments the image into a Voronoï region, $V_{r}$,

313 whose region is the set of point in the image closer to $L_{r}$ than any other localization, $L_{j}$, in $R$.

314 The RE of each Voronoï region $\left(V_{r}\right)$ is then computed by dividing observed occurrences of the

315 primary species $\left(\left(L_{p}\right)_{p \in P}\right)$ inside the region, where $P$ denotes the full set of indices belonging to

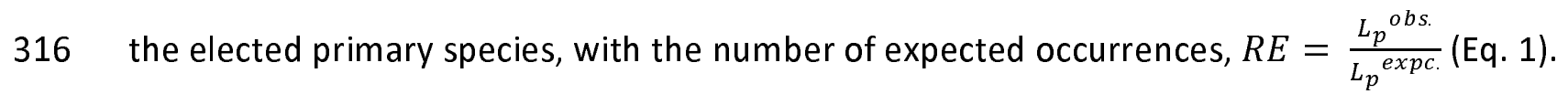

317 The number of expected occurrences is calculated by dividing the area of the region with the

318 total area analyzed and multiplying it by total number of primary localizations, $L_{p}{ }^{\text {expc. }}=$

$319 \frac{V_{r}^{\text {area }}}{\sum V_{r}^{\text {area }}}\left|L_{r}\right|$ (Eq. 2).

\section{Primary cultures}

321 Hippocampal neurons from E19 (embryonic) Wistar rat embryos of mixed gender (Charles

322 River, Wilmington, MA) were plated on $15 \mathrm{~mm}$ coverslips using 6 well plates in at a density of

323 approximately 100.000 cells/well. The coverslips were sterilized with UV and first coated with

324 poly-L-lysine (Gibco) overnight and then coated with Neurobasal media (Gibco) supplemented

325 with 4\% FBS (Gibco), 1:100 Glutamax (Gibco), 100U/mL penicillin, and 10mg/Ml streptomycin

326 (Invitrogen) overnight. The rat brains were removed and placed in ice-cold dissection media:

327 HBSS (Gibco) supplemented with 30mM glucose, 10mM HEPES (Gibco) (pH 7.4), 1mM sodium 
328 pyruvate (Gibco), $100 \mathrm{U} / \mathrm{mL}$ penicillin, and $10 \mathrm{mg} / \mathrm{mL}$ streptomycin (Sigma). The brains were cut

329 in half by sagittal incision. To access the hippocampus, each hemisphere has the medial section

330 facing up and the corpus callosum was removed. After isolation, the hippocampi were treated

331 with sterile filtered papain solution (Worthington) at $37 \mathrm{C}$ for $20 \mathrm{~min}$, triturated with two

332 different diameter fire-polished Pasteur pipettes 10 times each, and filtered through a 70 $\mu \mathrm{m}$

333 cell strainer to remove cell debris. Density was calculated by a hemocytometer. The cells were

334 seeded in Neurobasal media supplemented with 4\% FBS, 2\% B27 supplement (Gibco), 1:100

335 Glutamax (Gibco), 100U/mL penicillin, and $10 \mathrm{mg} / \mathrm{ml}$ streptomycin (Invitrogen). After $24 \mathrm{~h}$ of

336 seeding, the growth media was substituted with serum and glutamate free media and cultured

337 for 12-14 days in vitro (DIV). The cultures were stored at 35C with $5 \% \mathrm{CO} 2$. Media was changed

338 every 3-4 days by replenishing half the media with fresh growth media.

339 Heterologous cell cultures

340 PC-12 cells are derived from neuroendocrine chromaffin cells, and were maintained in

341 Dulbecco's Modified Eagle Medium with 10\% fetal bovine serum, 5\% horse serum and 1\%

342 penicillin/streptomycin.

\section{Immunocytochemistry}

344 The cell samples were fixed in paraformaldehyde (3\%) and washed three times in glycine (20

$345 \mathrm{mM})$ and $\mathrm{NH} 4 \mathrm{Cl}(50 \mathrm{mM})$ in PBS. Subsequently, the cells were washed in blocking buffer (5\%

346 Donkey serum, 1\% BSA in PBS), and incubated in blocking permeabilization buffer (blocking

347 buffer with saponin (0.2\%)). Primary antibody was applied in blocking buffer for $60 \mathrm{~min}$,

348 followed by 3-5 min incubation in blocking buffer. Secondary antibody was applied in blocking

349 buffer for $45 \mathrm{~min}$, and the sample was incubated $2 x$ for $5 \mathrm{~min}$ in blocking buffer. Samples were 
350 washed in PBS twice and post-fixated in paraformaldehyde (3\%) for $15 \mathrm{~min}$. Samples were

351 washed twice in glycine $(20 \mathrm{mM})$ and $\mathrm{NH} 4 \mathrm{Cl}(50 \mathrm{mM})$ in PBS and stored in PBS at $4{ }^{\circ} \mathrm{C}$ until

352 imaging

353 Antibodies

354 We used the following primary antibodies for staining: Anti-Stx1 at 1:500 (Synaptic Systems, 355110 011), anti-Munc18-1 at 1/500 (Synaptic Systems, 116 002), anti-hDAT at 1:1000 (Sigma-

356 Aldrich, MAB369), anti-hNET at 1:1000 (MAb Technologies, NET17-1) and anti-EEA1 at 1:1000

357 (Abcam, Ab2900). Seconday antibodies with conjugated AF568 or AF647 were added in 1:400

358 dilution.

359 dSTORM

360 For dSTORM we used a buffer containing $\beta$-mercaptoehtanol and an enzymatic oxygen

361 scavenger system (10\% (w/V) glucose, 1\% (V/V) beta-mercaptoethanol, $2 \mathrm{mM}$

362 cyclooctatetraene, $50 \mathrm{mM}$ Tris- $\mathrm{HCl}\left(\mathrm{pH}\right.$ 8), $10 \mathrm{mM} \mathrm{NaCl}, 34 \mu \mathrm{g} \mathrm{mL}{ }^{-1}$ catalase, $28 \mu \mathrm{g} \mathrm{m}^{-1}$ glucose

363 oxidase). The imaging was performed with an ECLIPSE Ti-E epifluoresence/TIRF microscope

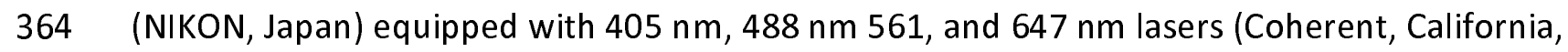

365 USA). All lasers were individually shuttered and collected in a single fiber to the sample through

366 a 1.49 NA, 100x, apochromat TIRF oil objective (NIKON). For dual-color dSTORM, we used a

367 dichroic mirror with the range 350-412, 485-490, 558-564, and 637-660 nm (97,335 QUAD C-

368 NSTORM C156921). The excitation light was filtered at the wavelengths: $401 \pm 24 \mathrm{~nm}, 488 \pm 15$

$369 \mathrm{~nm}, 561 \pm 15 \mathrm{~nm}, 647 \pm 24 \mathrm{~nm}$. The emitted light was filtered at the wavelengths: $425-475,505-$

$370545,578-625$, and $664-787 \mathrm{~nm}$, and secondly by an extra filter to decrease noise (561 nm

371 Longpass, Edge Basic, F76-561, AHF). A motorized piezo stage controlled by a near-infrared 
372 light-adjusted perfect focus system (NIKON) was applied to the system to reduce any sample

373 drift over time in the z-direction. Dual-color dSTORM images were constructed from 10,000

374 frames for each color, taken at a $16 \mathrm{~ms}$ frame rate, with each color alternating by frame.

375 Photons were collected with an iXon3 897 EM-CCD camera (Andor, United Kingdom). Laser

376 powers used were $2.3 \mathrm{~kW} \mathrm{~cm}^{2}$ for $647 \mathrm{~nm}, 1.0 \mathrm{~kW} \mathrm{~cm}$ for $488 \mathrm{~nm}$ and for $561 \mathrm{~nm}$. The $405 \mathrm{~nm}$

377 laser was used to incrementally increase blinking behavior at power $<0.1 \mathrm{~kW} \mathrm{~cm}{ }^{2}$. For 3D-

378 dSTORM, a cylindrical lens was placed before the camera to impart astigmatism. A reference z-

379 stack of fluorescent beads (TetraSpeck) was acquired with $25 \mathrm{~nm}$ intervals spanning $2 \mu \mathrm{m}$

380 before each imaging session. Localizations from dSTORM videos were fitted with 3D-

381 DAOSTORM [38], with a background sigma of 8, maximum likelihood estimation as the fitting

382 error model, 20 peak identification iterations, an initial sigma estimate of 1.5 and a threshold

383 adjusted to each imaging session. A linear fit in both the $x-z$ and $y-z$ plane was subtracted to

384 account for coverslip tilt.

385 Statistical Analysis.

386 Choice of statistical analysis is presented in the legends associated with each figure, and where

387 specified multiple testing was corrected for using the Bonferroni-Holmes correction. All $n$ -

388 values are individual images or simulations. Statistical analyses were carried out with the open-

389 source python packages SciPy v1.5.2, Numpy v1.18.1, and Seaborn v0.11.0, and linear models in

390 Statsmodels v0.12.2. Boxplots show $25^{\text {th }}$ and $75^{\text {th }}$ percentile, with whiskers indicating data

391 within 1.5 times the interquartile range. Remaining data were plotted as outliers. No statistical

392 methods were used to predetermine sample sizes. 
393 Data availability

394 The data presented in this study are available from the corresponding author upon reasonable 395 request.

396 Code Availability

397 All python functions and working examples with data from the simulated $2 \mathrm{D}$ and $3 \mathrm{D}$ vesicle

398 example are available online at: https://github.com/Ejdrup/relative-enrichment. The figure-

399 specific analyses are available from the corresponding author upon reasonable request.

400 Acknowledgements

401 The work was supported by the Lundbeck Foundation grants R266-2017-4331 (UG), R276-2018-

402792 (UG), R230-2016-3154 (M.D.L.) and R303-2018-3540 (F.H.).

\section{Author Contribution}

404 A.L.E. developed the method with help from M.D.L. and N.L. Experiments were performed by

405 A.L.E., N.L., M.D.L. and A.K.. Simulations and analysis were carried out by A.L.E. A.L.E. wrote the 406 manuscript. A.L.E., M.D.L., F.H.H., K.L.M. and U.G. edited the manuscript. All authors reviewed 407 and critically evaluated the manuscript. K.L.M. and U.G. supervised the project.

408 Competing interests

409 The authors declare no competing financial interests. 
1. Dunn, K.W., M.M. Kamocka, and J.H. McDonald, A practical guide to evaluating colocalization in biological microscopy. Am J Physiol Cell Physiol, 2011. 300(4): p. C72342.

2. Abbe, E., Beiträge zur Theorie des Mikroskops und der mikroskopischen Wahrnehmung. Archiv für Mikroskopische Anatomie, 1873. 9(1): p. 413-468.

3. Rust, M.J., M. Bates, and X. Zhuang, Sub-diffraction-limit imaging by stochastic optical reconstruction microscopy (STORM). Nat Methods, 2006. 3(10): p. 793-5.

4. Betzig, E., et al., Imaging intracellular fluorescent proteins at nanometer resolution. Science, 2006. 313(5793): p. 1642-5.

5. Folling, J., et al., Fluorescence nanoscopy by ground-state depletion and single-molecule return. Nat Methods, 2008. 5(11): p. 943-5.

6. Lang, T. and S.O. Rizzoli, Membrane protein clusters at nanoscale resolution: more than pretty pictures. Physiology (Bethesda), 2010. 25(2): p. 116-24.

7. Rahbek-Clemmensen, T., et al., Super-resolution microscopy reveals functional organization of dopamine transporters into cholesterol and neuronal activity-dependent nanodomains. Nat Commun, 2017. 8(1): p. 740.

8. Rossy, J., et al., Method for co-cluster analysis in multichannel single-molecule localisation data. Histochem Cell Biol, 2014. 141(6): p. 605-12.

9. Malkusch, S., et al., Coordinate-based colocalization analysis of single-molecule localization microscopy data. Histochem Cell Biol, 2012. 137(1): p. 1-10.

10. Pageon, S.V., et al., Clus-DoC: a combined cluster detection and colocalization analysis for single-molecule localization microscopy data. Mol Biol Cell, 2016. 27(22): p. 36273636.

11. Georgieva, M., et al., Nanometer resolved single-molecule colocalization of nuclear factors by two-color super resolution microscopy imaging. Methods, 2016. 105: p. 44-55.

12. Levet, F., et al., A tessellation-based colocalization analysis approach for single-molecule localization microscopy. Nat Commun, 2019. 10(1): p. 2379.

13. Dini, S., B.J. Binder, and J.E.F. Green, Understanding interactions between populations: Individual based modelling and quantification using pair correlation functions. J Theor Biol, 2018. 439: p. 50-64.

14. Tang, A.H., et al., A trans-synaptic nanocolumn aligns neurotransmitter release to receptors. Nature, 2016. 536(7615): p. 210-4.

15. Li, T.P., et al., Protein Crowding within the Postsynaptic Density Can Impede the Escape of Membrane Proteins. J Neurosci, 2016. 36(15): p. 4276-95.

16. Gronnier, J., et al., Structural basis for plant plasma membrane protein dynamics and organization into functional nanodomains. Elife, 2017. 6.

17. Heer, J. and M. Bostock, Crowdsourcing graphical perception: using mechanical turk to assess visualization design. $\mathrm{CHI}$ '10: Proceedings of the SIGCHI Conference on Human Factors in Computing Systems, 2010: p. 203-212.

18. Coltharp, C., X. Yang, and J. Xiao, Quantitative analysis of single-molecule superresolution images. Curr Op in Struct Biol, 2014. 28: p. 112-21. 
452 19. Werner, C., M. Sauer, and C. Geis, Super-resolving Microscopy in Neuroscience. Chem Rev, 2021. 121(19): p. 11971-12015.

20. Vardar, G., et al., Distinct Functions of Syntaxin-1 in Neuronal Maintenance, Synaptic Vesicle Docking, and Fusion in Mouse Neurons. J Neurosci, 2016. 36(30): p. 7911-24.

21. Stepien, K.P., E.A. Prinslow, and J. Rizo, Munc18-1 is crucial to overcome the inhibition of synaptic vesicle fusion by alphaSNAP. Nat Commun, 2019. 10(1): p. 4326.

22. Padmanabhan, P., et al., Need for speed: Super-resolving the dynamic nanoclustering of syntaxin-1 at exocytic fusion sites. Neuropharmacology, 2020. 169: p. 107554.

23. Lee, S., et al., Munc18-1 induces conformational changes of syntaxin-1 in multiple intermediates for SNARE assembly. Sci Rep, 2020. 10(1): p. 11623.

24. Pertsinidis, A., et al., Ultrahigh-resolution imaging reveals formation of neuronal SNARE/Munc18 complexes in situ. Proc Natl Acad Sci U S A, 2013. 110(30): p. E2812-20.

25. Kavanagh, D.M., et al., A molecular toggle after exocytosis sequesters the presynaptic syntaxin 1a molecules involved in prior vesicle fusion. Nat Commun, 2014. 5(1): p. 5774.

26. Heilemann, M., et al., Subdiffraction-resolution fluorescence imaging with conventional fluorescent probes. Angew Chem Int Ed Engl, 2008. 47(33): p. 6172-6.

27. Bar-On, D., et al., Super-resolution imaging reveals the internal architecture of nanosized syntaxin clusters. J Biol Chem, 2012. 287(32): p. 27158-67.

28. Lycas, M.D., et al., Nanoscopic dopamine transporter distribution and conformation are inversely regulated by excitatory drive and D2-autoreceptor activity. bioRxiv, 2021.

29. Cragg, S.J. and M.E. Rice, DAncing past the DAT at a DA synapse. Trends in Neurosciences, 2004. 27(5): p. 270-277.

30. Condon, M.D., et al., Plasticity in striatal dopamine release is governed by releaseindependent depression and the dopamine transporter. Nat Commun, 2019. 10(1): p. 4263.

31. Vuorenpaa, A., et al., Differential Internalization Rates and Postendocytic Sorting of the Norepinephrine and Dopamine Transporters Are Controlled by Structural Elements in the N Termini. J Biol Chem, 2016. 291(11): p. 5634-5651.

32. Block, E.R., et al., Brain Region-Specific Trafficking of the Dopamine Transporter. J Neurosci, 2015. 35(37): p. 12845-58.

33. Matthies, H.J., et al., Subcellular localization of the antidepressant-sensitive norepinephrine transporter. BMC Neurosci, 2009. 10: p. 65.

34. Gruenberg, J., G. Griffiths, and K.E. Howell, Characterization of the early endosome and putative endocytic carrier vesicles in vivo and with an assay of vesicle fusion in vitro. J Cell Biol, 1989. 108(4): p. 1301-16.

35. Jovic, M., et al., The early endosome: a busy sorting station for proteins at the crossroads. Histol Histopathol, 2010. 25(1): p. 99-112.

36. Rahbek-Clemmensen, T., et al., Super-resolution microscopy reveals functional organization of dopamine transporters into cholesterol and neuronal activity-dependent nanodomains. Nature Communications, 2017. 8(1): p. 1-14. Localization Microscopy Cluster Analysis and Quantification Methods. Patterns (N Y), 
495 38. Babcock, H., Y.M. Sigal, and X. Zhuang, A high-density 3D localization algorithm for 496 stochastic optical reconstruction microscopy. Opt Nanoscopy, 2012. 1(6): p. 1-10.

497 39. Marin, Z., et al., PYMEVisualize: an open-source tool for exploring 3D super-resolution 498 data. Nat Methods, 2021. 18(6): p. 582-584.

499 40. Barna, L., et al., Correlated confocal and super-resolution imaging by VividSTORM. Nat $500 \quad$ Protoc, 2016. 11(1): p. 163-83.

501 41. Nehme, E., et al., DeepSTORM3D: dense 3D localization microscopy and PSF design by

502

503 504 deep learning. Nat Methods, 2020. 17(7): p. 734-740.

42. Prokop, S., et al., PharmacoSTORM nanoscale pharmacology reveals cariprazine binding on Islands of Calleja granule cells. Nat Commun, 2021. 12(1): p. 6505.

505

506 
A

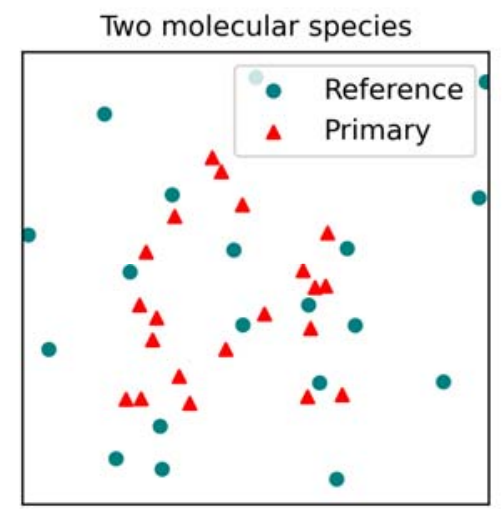

D

Calculation of RE score for single reference region:

(Eq. 2) $p^{\exp }=\frac{0.24 \mu m^{2}}{1.3 \mu m^{2}} 20$ locs. $=3.7$ locs.

(Eq. 1) $R E$ score $=\frac{1 \text { locs. }}{3.7 \text { locs. }}=0.27$
B

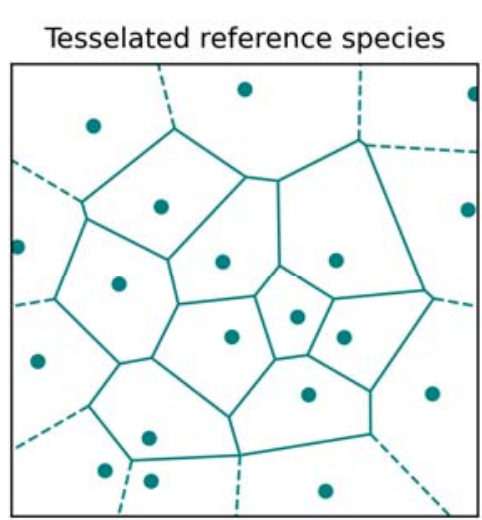

C Superimposed primary species

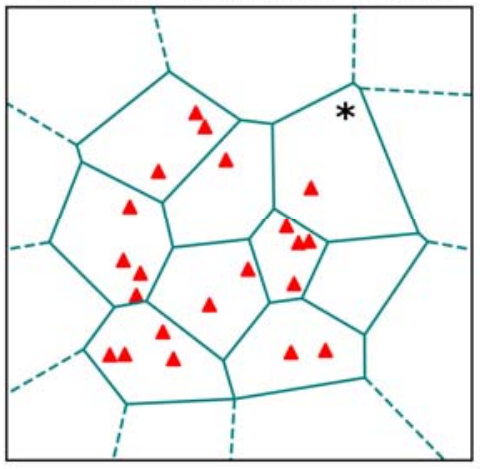

$\mathrm{E}$

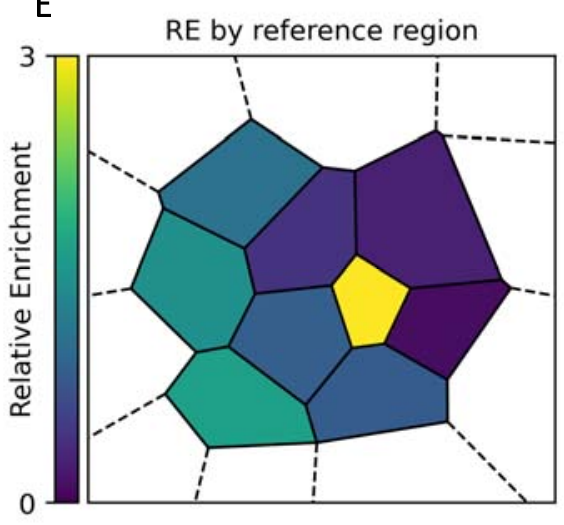

Figure 1. Tessellation-based assessment of relative enrichment.

512 (A) Simulated random distribution of two molecular species. For computation of relative

513 enrichment (RE) one is assigned as the primary species and one as the reference species. RE can

514 be bidirectionally computed, and will by design yield different results if the two species are

515 switched.

516 (B) Voronoï-regions from tessellation of the reference species from (A).

(C) Primary species superimposed on the Voronoï-regions of the reference species.

518 (D) Calculation of single RE value from Voronoï-region marked with $\left({ }^{*}\right)$ in $(C)$, based on the 
bioRxiv preprint doi: https://doi.org/10.1101/2022.02.08.479535; this version posted February 9, 2022. The copyright holder for this preprint (which was not certified by peer review) is the author/funder. All rights reserved. No reuse allowed without permission.

519 distribution of the primary species.

520 (E) Voronoï-regions for the reference species, color-coded by their individual RE scores. All

521 calculated as in panel (D).

522 


\section{Figure 2}

A

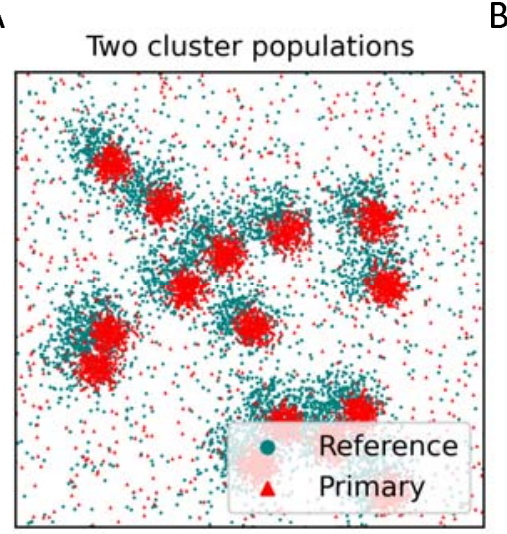

D

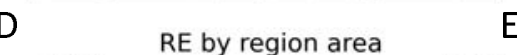

B

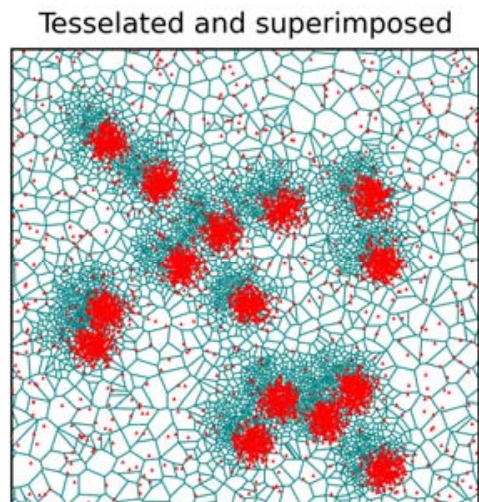

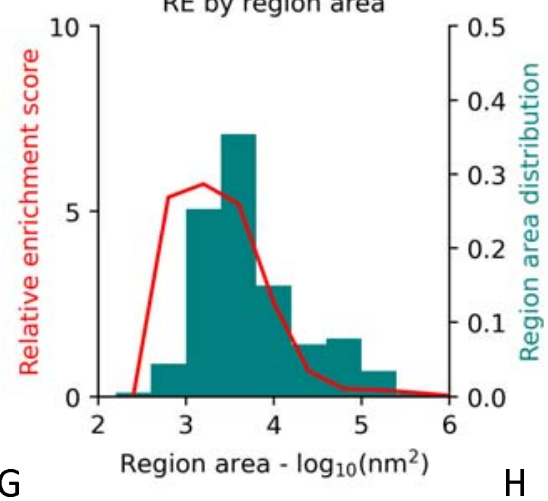

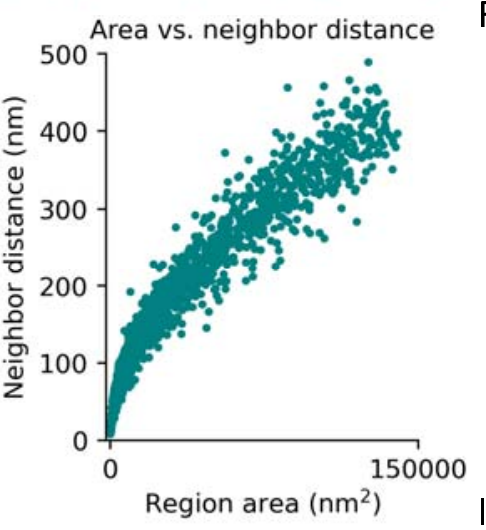

C

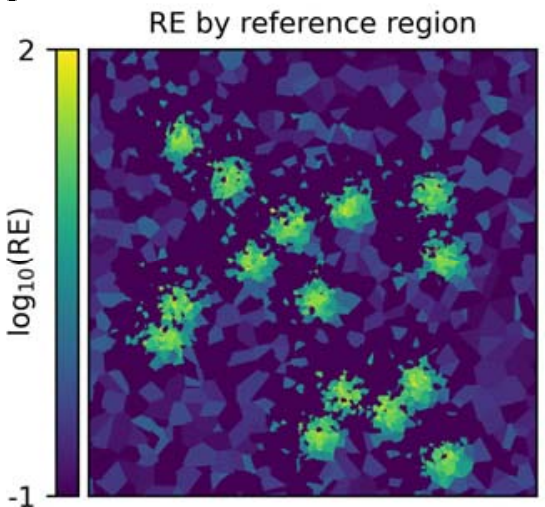

F RE by neighbor distance

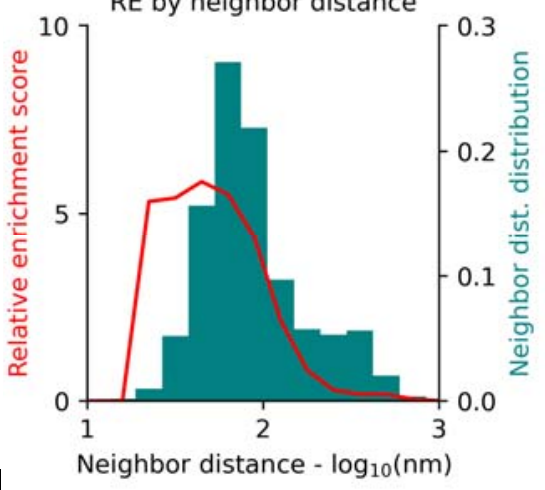

RE by neighbor distance
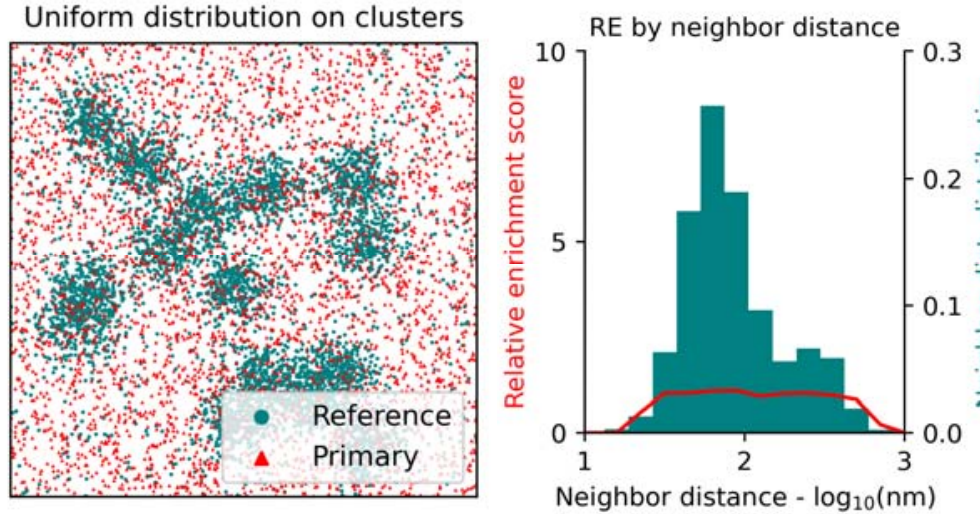

RE by reference region

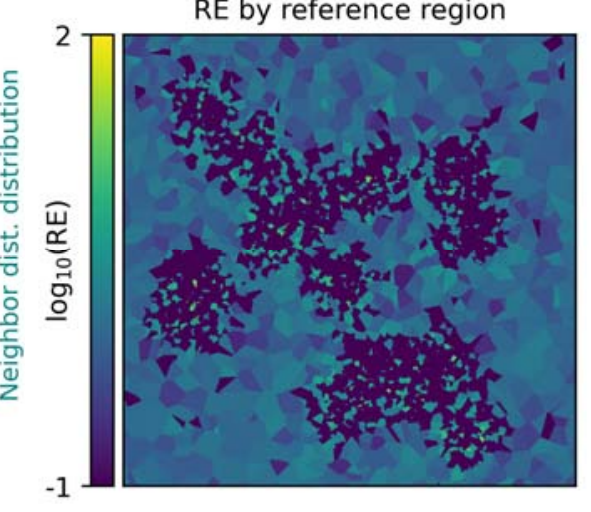

Figure 2. Visualising RE across densities for larger images.

526 (A) Simulation of two colocalizing populations of normally-distributed clusters with random

527 background noise. One species of clusters is slightly off-set from the other.

528 (B) Voronoï-regions of the reference species from (A), with the primary species as overlay.

529 (C) Voronoï regions for the reference species, color-coded by their individual RE scores, 
530 calculated based on the distribution of the primary species.

531 (D) Mean RE score across reference densities. Reference regions are binned by area, and mean

532 RE score calculated for each bin. RE score plotted on left-hand y-axis, and relative bin

533 distribution plotted on right-hand $\mathrm{y}$-axis.

534 (E) Comparison of region area versus mean distance to nearest neighbours for the reference

535 species.

536 (F) Mean RE score across reference densities, binned by nearest neighbour distance (NND),

537 rather than area.

538 (G) Simulation of two protein populations: a clustered and a uniform distribution.

539 (H) Same plot as (F), but for the uniform primary species distribution data shown on (G).

540 (I) Same plot as (C), but for the uniform primary species distribution data shown on (G). 


\section{Figure 3}

A

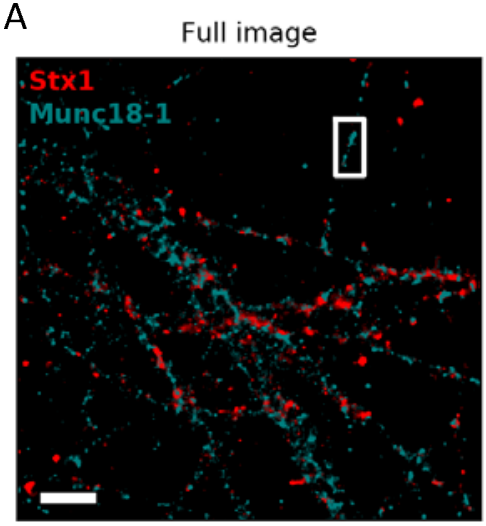

D

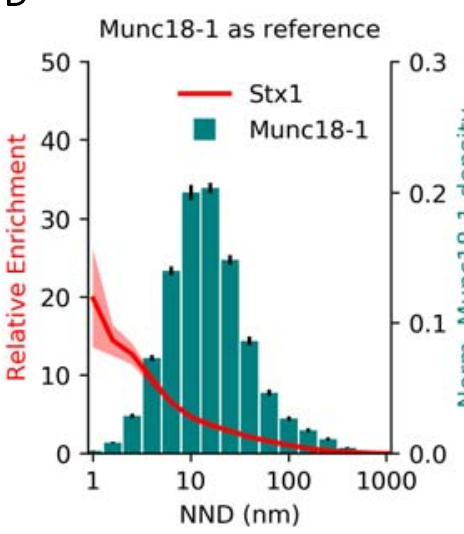

G
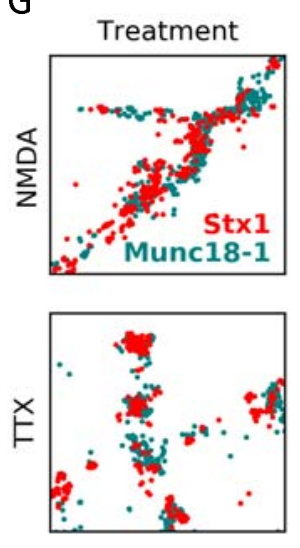

$\mathrm{H}$
B

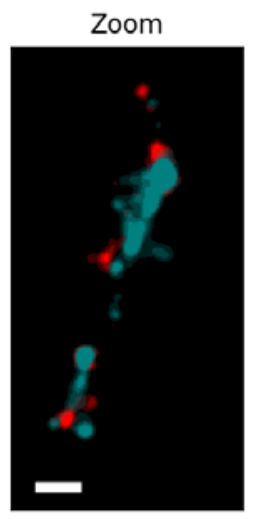

E

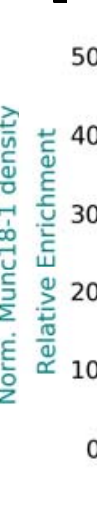

Stx1 as reference
Localizations

C ${ }_{\text {Munc18-1 }}^{\text {Reference }}$ Stx1
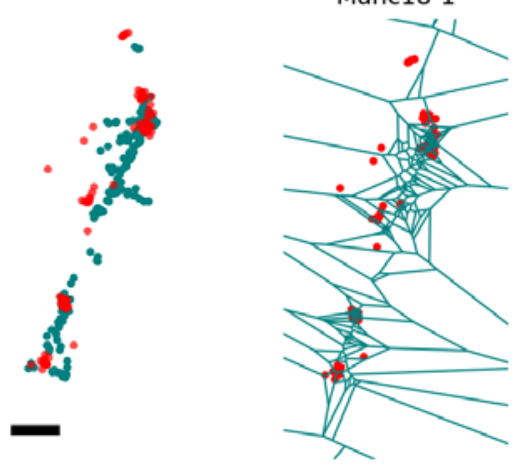

$\mathrm{F}$

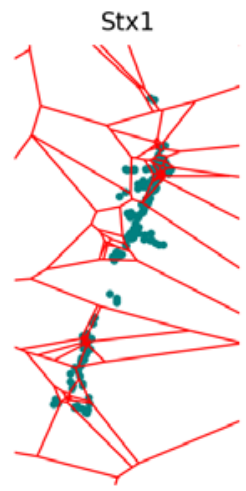

Reference

Munc18-1 Stx
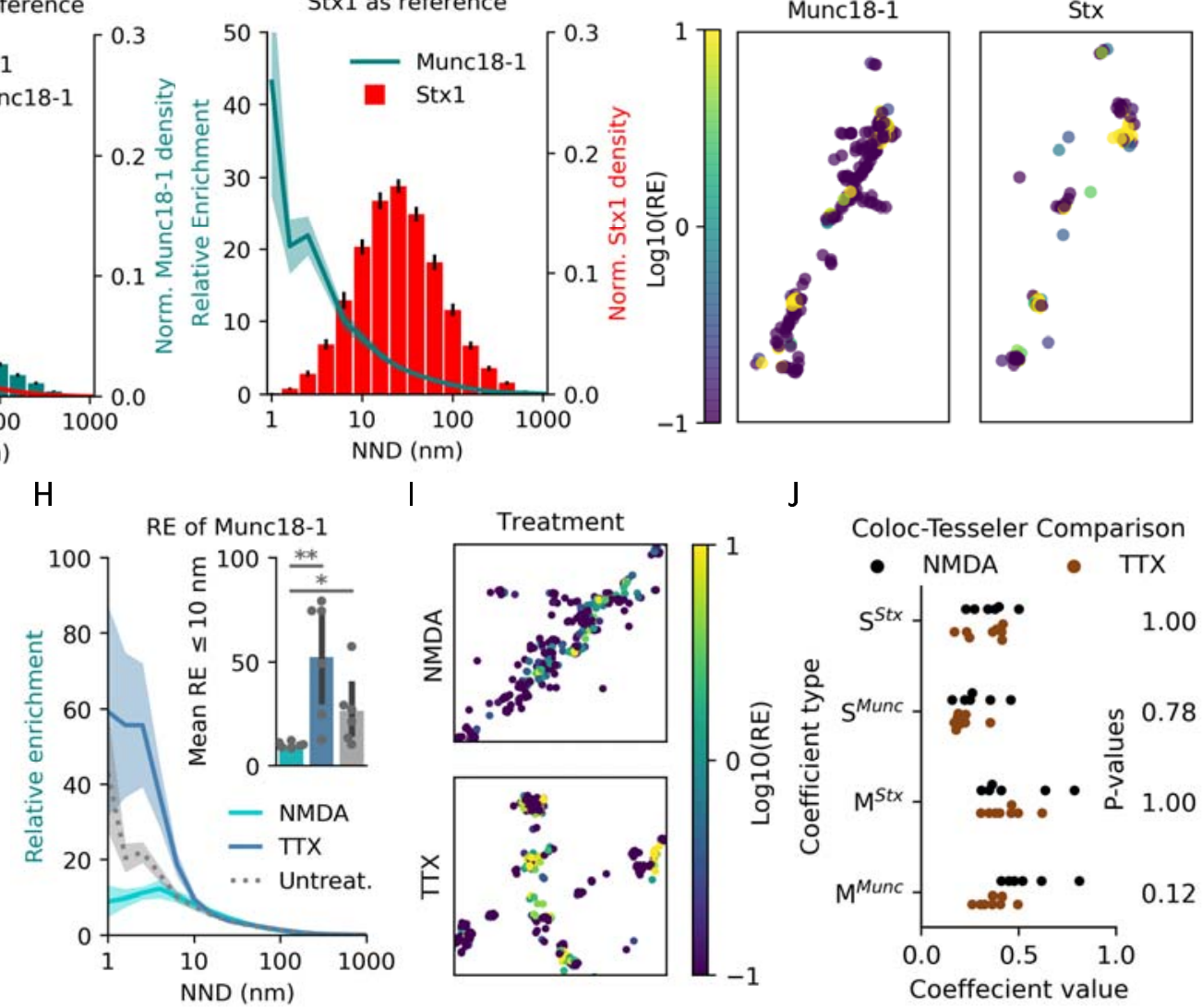

I

Treatmen

J
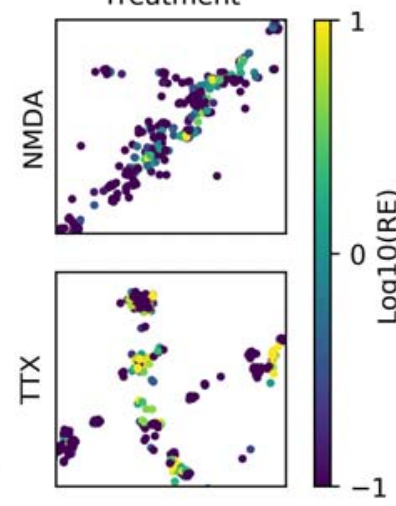

Coloc-Tesseler Comparison
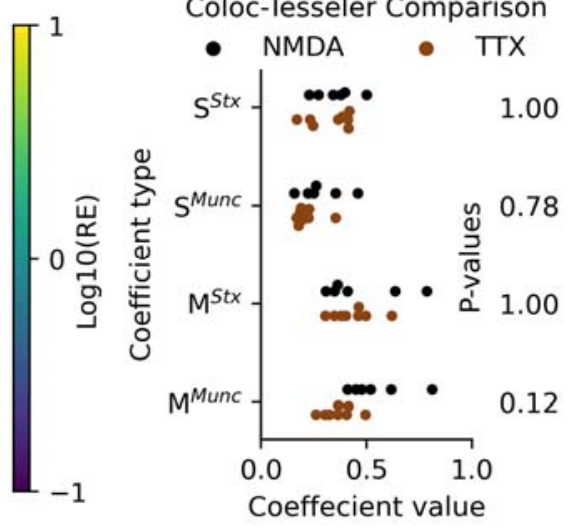

544 Figure 3. Visualising RE across densities for larger images.

545 (A) Representative image of Stx1 (red) and Munc18-1 (teal) in primary hippocampal neurons

546 acquired with dSTORM. Molecular localizations visualized by gaussian representation. Scale bar

547 is $4 \mu \mathrm{m}$. 
548 (B) Zoom on single varicosity from (A), with both Gaussian representation (left) and individual

549 localizations (right). Scale bars are $400 \mathrm{~nm}$.

550 (C) Voronoï-regions for tessellation of either Munc18-1 (left) or Stx1 (right). Individual

551 localizations of opposite species are plotted beneath. Same scale as (B).

552 (D) Munc18-1 regions binned by nearest neighbour distance, with mean Stx1 RE value for each

553 bin as line plot. RE score on left-hand y-axis, and relative bin distribution on right-hand y-axis.

554 Shaded area and black bars indicate S.E.M., $\mathrm{n}=8$ images.

555 (E) Same as (D), but with reference and primary species reversed.

556 (F) Localizations of the reference species color-coded by RE score for Munc18-1 (left) and Stx1

557 (right).

558 (G) Representative axons of hippocampal neurons after treatment with NMDA (top) or TTX

559 (bottom).

560 (H) Relative enrichment of Munc18-1 across Stx1 densities after NMDA (dashed, $n=7$ images)

561 or TTX (dotted, $\mathrm{n}=8$ images) treatment. Shaded area indicates S.E.M.. Inset: Mean RE of

562 regions with an NND $\leq 10 \mathrm{~nm}$ for NMDA, $\Pi \mathrm{TX}$ or untreated cultures (Untreat:NMDA., $\mathrm{p}=0.004$;

563 Untreat:TTX, $p=0.084 ;$ NMDA:TTX, $p=0.039$. Unpaired, two-tailed student's t-test by image,

564 FWER correction with Bonferroni-Holm).

565 (I) Images from (G), with Stx1 localizations color-coded by RE of Munc18-1.

566 (J) Spearman (S) and Mander's (M) coefficient computed as per [12] $\left(H_{0}:\right.$ NMDA $=$ TTX. $S^{\text {Stx }}, p=$

567 1.00; $S^{\text {Munc }}, p=0.78 ; M^{S t x}, p=1.00 ; M^{M u n c}, p=0.12$. Unpaired, two-tailed student's t-test by

568 image, FWER correction with Bonferroni-Holm). 
570 Figure 4

A

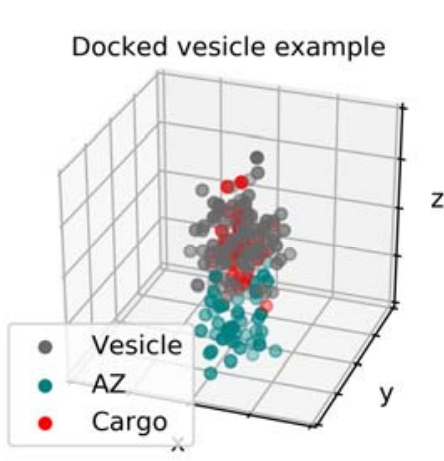

D

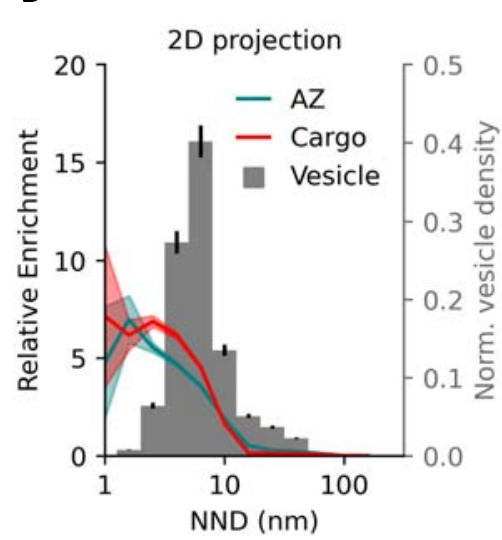

B

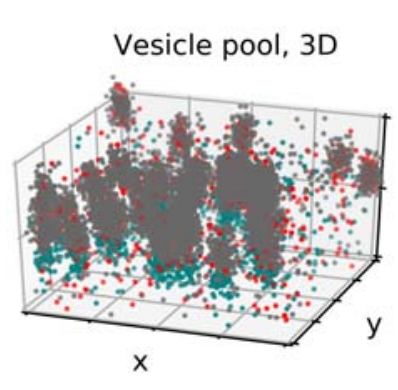

E

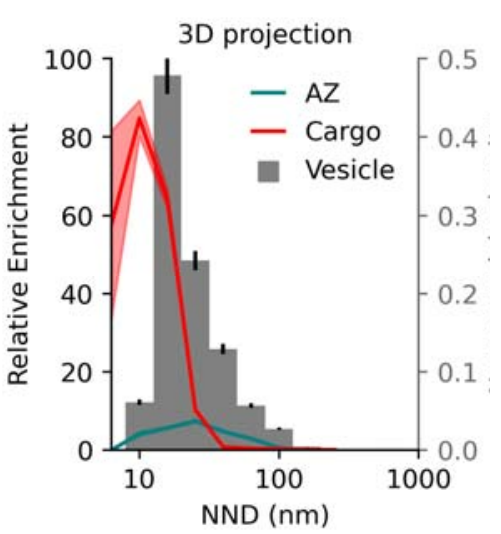

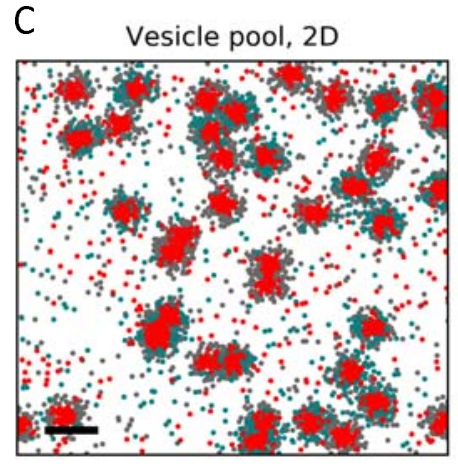

$\mathrm{F}$

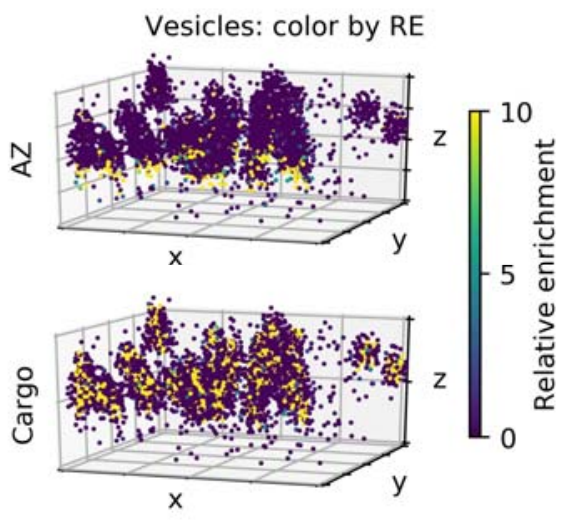

571

573 Figure 4. Validation of RE-scoring in 3D.

574 (A) Simulation of a single docked vesicle with three molecular species: a vesicle membrane-

575 bound, a SNARE-like and a luminal. Grid is 50 by $50 \mathrm{~nm}$.

576 (B) Representative simulation of twenty vesicles. Half docked at the membrane and associated

577 with SNARE-like molecules, and half in the intracellular space without SNARE-association. Grid

578 is 200 by $200 \mathrm{~nm}$.

579 (C) 2 D projection of representative simulation in (B). Scale bar is $100 \mathrm{~nm}$.

580 (D) Vesicle Voronoï regions binned by nearest neighbour distance in 2D, with mean RE value of

581 each bin for both SNARE-like (blue) and luminal (green) as line plot. Shaded area indicates 
582 S.E.M., $\mathrm{n}=20$. Scale bar is $100 \mathrm{~nm}$.

583 (E) Vesicle Voronoï regions binned by nearest neighbour distance in 3D, with mean RE value of

584 each bin for both SNARE-like (blue) and luminal (green) as line plot. Shaded area indicates

585 S.E.M., $n=20$.

586 (F) Simulated vesicle localizations from (B) color-coded for their relative enrichment of either

587 SNARE (top) for luminal (bottom) localizations. Grid is 200 by $200 \mathrm{~nm}$. 

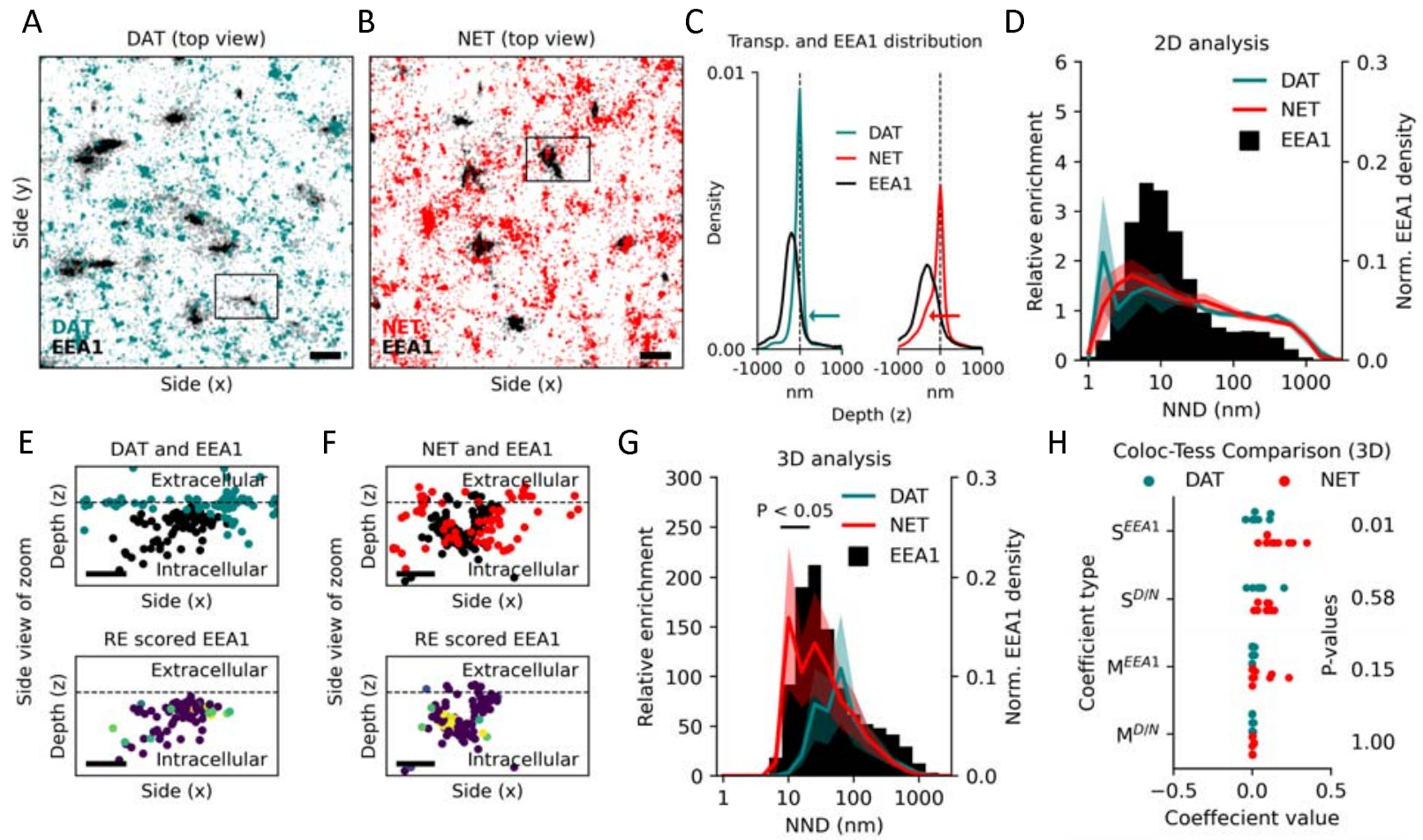

590

Figure 5. Neurotransmitter transporter localization to early endosomes.

592 (A) Representative top view of image with EEA1 (black) and DAT (teal) in PC-12 cells acquired

593 with astigmatic dSTORM. Molecular localizations visualized by gaussian representation. Scale

594 bar is $0.5 \mu \mathrm{m}$.

595 (B) Representative top view of image with EEA1 (black) and NET (red) in PC-12 cells acquired

596 with astigmatic dSTORM. Molecular localizations visualized by gaussian representation. Scale

597 bar is $0.5 \mu \mathrm{m}$.

598 (C) Mean distribution of transporter and EEA1-positive structure depth. Normalized to peak

599 transporter depth. Arrows indicate the differential intracellular accumulation of NET.

600

(D) EEA1 Voronoï regions binned by nearest neighbour distance in 2D, with mean RE value of

601 each bin for both DAT (teal, $n=8$ images) and NET (red, $n=9$ images) as dots. Error bars area 
602 indicates S.E.M..

603 (E, F) Side view of black box from A and B. Top panel shows both species, and bottom panel

604 EEA1 localization color-coded by RE score. Dashed lines indicate plasma membrane depth as

605 assessed by median transporter location in z. Scale bar is $200 \mathrm{~nm}$.

$606(G)$ Same as (D), but assessed in 3D $\left(H_{0}\right.$ : DAT = NET. Black line indicates bins with $p<0.05$.

607 Unpaired, one-sided student's t-test by image)

608 (H) Spearman (S) and Mander's (M) coefficient computed in 3D as per [12] $\left(H_{0}\right.$ : DAT = NET. $S^{\text {EEA1 }}$,

$609 p=0.01 ; S^{D / N}, p=0.58 ; M^{E E A 1}, p=0.15 ; M^{D / N}, p=1.00$. Unpaired, one-sided student's t-test by

610 image, FWER correction with Bonferroni-Holm). 